\title{
A preliminary study of the clinical significance of folate receptor- positive circulating tumor cell in the management of hepatobiliary- pancreatic cancers
}

\author{
Yong-gang $\mathrm{He}^{1}$, Lu Zheng ${ }^{1}$, Ming-fa Gao ${ }^{1}$, Yi-chen Tang ${ }^{1}$, Yu-ming Li ${ }^{1}$, Kai-huan $\mathrm{Yu}^{2}$, Jing $\mathrm{Li}^{1}$, \\ Xiao-bing Huang ${ }^{1}$ \\ ${ }^{1}$ Department of Hepatobiliary Surgery, The Second Affiliated Hospital of Army Medical University, Chongqing, China; ${ }^{2}$ Department of \\ Hepatobiliary Surgery, Renmin Hospital of Wuhan University, Wuhan, China \\ Contributions: (I) Conception and design: YG He, J Li, XB Huang; (II) Administrative support: YC Tang, XB Huang, J Li; (III) Provision of \\ study materials or patients: YG He, MF Gao, YM Li, KH Yu; (IV) Collection and assembly of data: L Zheng, YC Tang; (V) Data analysis and \\ interpretation: YG He, L Zheng; (VI) Manuscript writing: All authors; (VII) Final approval of manuscript: All authors. \\ Correspondence to: Xiao-bing Huang; Jing Li. Department of Hepatobiliary Surgery, The Second Affiliated Hospital of Army Medical University, No. \\ 183, Xinqiaozheng Street, Shapingba District, Chongqing 400037, China. Email: 1038915320@qq.com; xqyylijing@163.com.
}

\begin{abstract}
Background: Hepatobiliary-pancreatic cancers (HBPs) are highly lethal, partly because of their usually late diagnosis. This multi-center, observational study aimed to explore the clinical significance of folate receptor-positive circulating tumor cell (FR+CTC) as a liquid biopsy approach in the differential diagnosis and management of HBPs.

Methods: We recruited 119 patients suspicious for HBPs and 60 cancer-free healthy individuals in the present study. Patients without definitive pathological assessment or without pre-operative FR+CTC analysis were excluded. FR+CTC was tested prior to surgery or tissue biopsy using the CytoploRare ${ }^{\circledR}$ Detection Kit. Serum biomarkers, including CA 125, CA 19-9, and CEA, were tested in selected patients. Post-operative FR+CTC analysis was also performed in a subset of the patients receiving surgical resection.

Results: With $8.65 \mathrm{FU} / 3 \mathrm{~mL}$ as the cut-off value, the sensitivity and specificity of FR+CTC in differential diagnosis were $98.1 \%$ and $79.1 \%$, respectively. The detection rate of FR+CTC was superior to conventional serum biomarkers (CA 19-9 > CA 125 > CEA). For the 16patients with matched post-operative FR+CTC analysis, FR+CTC levels significantly reduced after surgery $(\mathrm{P}=0.0084)$.

Conclusions: Our results demonstrated that FR+CTC analysis could be an efficacious non-invasive biomarker in differential diagnosis and surveillance of HBPs, though further investigation with a larger sample size is required.
\end{abstract}

Keywords: Circulating tumor cell (CTC); folate receptor; hepatobiliary cancer; pancreatic cancer; surgery

Submitted May 26, 2020. Accepted for publication Oct 21, 2020.

doi: $10.21037 /$ tcr-20-2192

View this article at: http://dx.doi.org/10.21037/tcr-20-2192

\section{Introduction}

Hepatobiliary-pancreatic cancers (HBPs) account for 1,519,000 of new cancer incidences and 1,379,000 of cancer deaths each year (1). The 5-year survival rate of hepatobiliary cancers (18\%), particularly pancreatic cancer $(8 \%)$, is dismal (2). Patients eligible for surgical resection have better prognosis. The most effective treatments for hepatocellular carcinoma (HCC) are liver resection and transplantation $(3,4)$. Also, radical resection is the only way to completely cure cholangiocarcinoma (5). For pancreatic cancers, surgery is the only proven effective treatment yet it only improves the 5-year survival rate to $15-20 \%$, and patients ineligible for surgery have a median survival of 
only 18 months (6). As the prognosis of HBPs depends largely on the tumor stage, early detection of HBPs is critical to improve the outcome. However, because of the asymptomatic nature of HBPs and lack of serum biomarkers with high diagnostic sensitivity and specificity, most of the patients are diagnosed at an advanced stage and lose the chance of surgical resection. Late diagnosis and lack of effective treatment options are considered to be the main causes of the high mortality in HBPs. For patients with hepatobiliary cancers, $18 \%$ already had distant metastasis at initial diagnosis. More specifically, in pancreatic cancer, such a proportion can be as high as $52 \%$ (2). In addition, patients with HBPs suffer from a high rate of relapse after surgical resection $(7,8)$. To increase the rate of identifying resectable HBPs and to facilitate prompt treatment of recurrent cancers, it is important to establish a non-invasive biomarker for differential diagnosis and surveillance of HBPs.

Carbohydrate antigen 125 (CA 125), carbohydrate antigen 19-9 (CA 19-9), and carcinoembryonic antigen (CEA) are widely used in clinical practice of HBPs. However, the utilities of these biomarkers in early diagnosis of HBPs are limited because they lack sufficient sensitivity in early stage malignancy while at the same time elevated level of these biomarkers are often seen in patients with certain benign hepatobiliary-pancreatic diseases $(3,5,9)$. Detection of circulating tumor cell (CTC), as a liquid biopsy technique, could be a possible solution for such problems. In various studies, CTCs have been shown to be present and detectable in early-stage lung cancer, gastric cancer, colorectal cancer, and a few other cancer types $(10,11)$. Of a variety of existing CTC detection technologies, the one that quantitatively assesses folate receptor (FR) expression for CTC identification appears most promising. FRs are cysteine-rich cell-surface glycoproteins, which can bind folate with high affinity to mediate cellular uptake of folate (12). FRs are found to be over expressed in a variety of cancer tissues, whereas most normal tissues and other cells in the circulatory system express these receptors at low to negligible levels (13-18). FR thus is a promising target for CTC detection.

Several studies had confirmed the diagnostic efficiency of FR+CTC in lung cancer, bladder cancer, pancreatic cancer, and colorectal cancer (19-24). FR+CTC has also been shown to be prognostic of chemotherapy response in patients with small cell lung cancer (20,25-27). In particular, a number of previous studies demonstrated that the enumeration of FR+CTC based on negative depletion and ligand-targeted PCR method has high sensitivity (73-82.5\%) and specificity (82-93\%) in lung cancer diagnosis (28-31). The aforementioned method first enriches CTCs through lysis of erythrocytes followed by leukocytes depletion. The first step, commonly referred to as "negative enrichment", ensures a wide variety of CTCs to be retained at the enrichment stage. Compared to antigen-dependent positive-enrichment techniques such as CellSearch $^{\circledR}$, negative enrichment techniques can lead to a lower false-negative rate. Next, CTCs are detected using the ligand-targeted PCR method, in which folate receptor alpha (FR $\alpha$ )-expressing CTCs are labelled with a detection probe consisting of an FR $\alpha$-targeting folic acid ligand and a specially designed oligonucleotide. PCR is performed to amplify the oligonucleotide bound to the FR $\alpha$ on the CTCs and enhance the detection sensitivity (18). Although $\mathrm{FR} \alpha$ was also found to be expressed in tumor tissues of hepatobiliary cancers and pancreatic cancers (32-36), the clinical utility of FR+CTC in these types of cancer remains to be examined. Hence, in this preliminary study, we aimed to explore the clinical significance of $\mathrm{FR}+\mathrm{CTC}$ in the differential diagnosis and management of HBPs.

We present the following article in accordance with the STARD reporting checklist (available at http://dx.doi. org/10.21037/tcr-20-2192).

\section{Methods}

\section{Study design}

This is a preliminary, multi-center, observational study conducted at the Xinqiao Hospital, Army Medical University and Renmin Hospital of Wuhan University from March 2017 to November 2019. A total of 119 patients suspected for HBPs and 60 cancer-free healthy individuals were recruited in this study. The detailed procedure for patient enrollment was shown in Figure 1. For the malignant group, the inclusion criteria were: (I) suspected for HBPs; (II) treatment-naïve and expected to undergo surgical resection or tissue biopsy; and (III) written informed consent obtained to have peripheral blood collected for FR+CTC analysis. Exclusion criteria were: (I) pathologically diagnosed as benign diseases or indeterminate malignancy; and (II) insufficient pre-operative peripheral blood sample $(<3 \mathrm{~mL})$ for FR+CTC analysis. The control group consisted of patients with pathologically confirmed benign diseases and healthy individuals who were systematically evaluated to be cancer-free in a health check-up performed within 


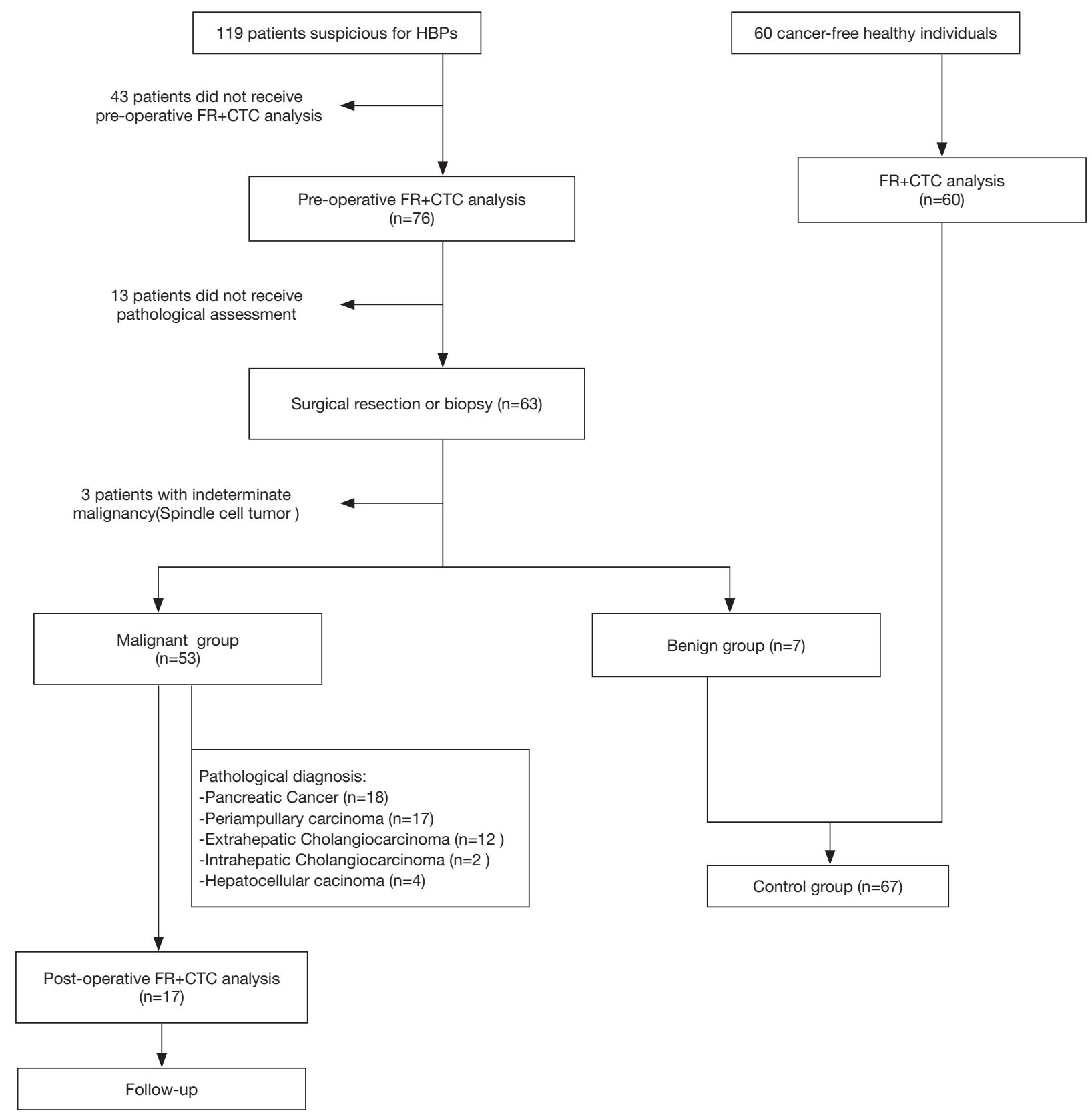

Figure 1 Flow diagram of patient enrollment.

the last six months. Except for healthy individuals, all participants received an abdominal computed tomography scan before surgery to assess the respectability. Tumor tissue specimens obtained during surgery were analyzed by an experienced pathologist using $\mathrm{H} \& \mathrm{E}$ staining method. Smear of bile was collected from the participants who did not undergo surgery for pathological assessment. Clinical staging and pathological subtyping were determined in accordance with the AJCC Cancer Staging system $\left(8^{\text {th }}\right.$ edition) and the respective requirements established by each participating hospital. The Institutional Review Board of each participating hospital has approved the study protocol (AF/SC-08/1.0). Written informed consent was obtained from each participant prior to the enrollment in to the study. The study was conducted in accordance with the Declaration of Helsinki (as revised in 2013). 


\section{Enumeration of FR+CTC}

Detection of FR+CTC was performed using the CytoploRare ${ }^{\circledR}$ Detection Kit provided by GenoSaber Biotech Co. Ltd. (Shanghai, China) as previously described (28). Three milliliters of peripheral blood were withdrawn into an EDTA-containing anti-coagulant tube from each participant. Pre-operative blood samples were collected within two weeks before surgery and post-operative blood samples were collected within two months after surgery. Rare FR+CTCs were first enriched by negative depletion method, in which red and white blood cells present in the blood sample were sequentially removed, first by a lysing buffer and then by a combination of anti-CD45 and antiCD14 immuno-magnetic beads. The enriched FR+CTCs were then labelled by an FR $\alpha$-targeting oligonucleotide probe. Using the proprietary ligand-targeted PCR method, FR+CTCs were enumerated by quantitative PCR (18). A serial of standard calibrators containing oligonucleotides $\left(10^{-14}\right.$ to $10^{-9} \mathrm{M}$, corresponding to 2 to $2 \times 10^{5} \mathrm{FU} / 3 \mathrm{~mL}$ blood) were used to create a standard curve for FR+CTC quantification. Folate receptor Unit (FU) per $3 \mathrm{~mL}$ of peripheral blood calculated from the standard curve was used to represent the FR+CTC level in each sample.

\section{Serum biomarker test}

An additional $3 \mathrm{~mL}$ of peripheral blood was withdrawn into a blood coagulation tube. After centrifuging at 3,500 rpm for $10 \mathrm{~min}$, the upper serum was collected for analysis. Serum biomarkers (including CA 125, CA 19-9, and CEA) were analyzed by radioimmunoassay (Abbott Lab., Chicago, IL, USA). The normal reference ranges were: CA $125<35 \mathrm{U} / \mathrm{mL}$, CA $19-9<37 \mathrm{U} / \mathrm{mL}$, and CEA $<5 \mathrm{ng} / \mathrm{mL}$.

\section{Statistical analysis}

Data analysis was performed using the Prism 5.0 software (GraphPad, CA, USA). Countable variables were expressed as number (percentage). Age and FR+CTC levels were expressed as median (range). Maximum tumor diameter was expressed as mean \pm standard deviation. Pre-operative FR+CTC levels between groups were compared using Man Whitney $U$ test. Dynamic changes in FR+CTC levels were compared using Wilcoxon signed rank test. The percentage change in FR+CTC level was calculated by "(Postoperative FR+CTC level - Pre-operative FR+CTC level)/Preoperative FR+CTC level $\times 100 \%$ ". A P value of less than 0.05 was considered to be statistically significant. The receiver operating characteristic (ROC) curve was plotted with sensitivity as the $y$-axis and 1-specificity as the $\mathrm{x}$-axis, and the area under the ROC curves (AUCs) were calculated for each threshold value to examine the diagnostic efficiency. The Youden index, calculated as sensitivity + specificity 1 , was used to evaluate the diagnostic efficiency for each threshold value. The one that maximized the Youden index was chosen as the optimal cut-off point.

\section{Results}

\section{Patients' demographics}

A total of 119 patients suspected for HBPs were recruited in this study (Figure 1). After excluding 43 patients who did not receive pre-operative $\mathrm{FR}+\mathrm{CTC}$ analysis, 12 patients who refused to undergo surgery or tissue biopsy, and 4 patients with indeterminate malignancy after surgery, 60 patients with pathologically confirmed HBPs were included in the malignant group. 7 patients diagnosed as having benign diseases after surgery together with 60 cancer-free healthy individuals were included in the control group. Baseline characteristics of the enrolled patients were summarized in Table 1 .

\section{Expression level of FR+CTC}

The median FR+CTC level of the malignant group was significantly higher than that of the control group (14.8 versus $7.5 \mathrm{FU} / 3 \mathrm{~mL}, \mathrm{P}<0.0001$, Figure $2 A$ ). ROC analysis indicated that the sensitivity and specificity of FR+CTC in the differential diagnosis of HBPs were $98.1 \%$ and $79.1 \%$, respectively, when $8.65 \mathrm{FU} / 3 \mathrm{~mL}$ was chosen as the cut-off value [area under curve $=0.945,95 \%$ confidence interval (CI): 0.905 0.986]. The false-positive rate of FR+CTC in the control group was $20.9 \%$. Notably, 6 of the 7 patients diagnosed as having benign inflammatory diseases had a false-positive FR+CTC result (chronic hepatitis: $11.7 \mathrm{FU} / 3 \mathrm{~mL}$; chronic cholecystitis: 23.8, 10.3 FU/3 mL; chronic choledochitis: $8.9 \mathrm{FU} / 3 \mathrm{~mL}$; serous adenoma of the pancreas: $10.5 \mathrm{FU} / 3 \mathrm{~mL}$; duodenal tubular adenoma: $11.2 \mathrm{FU} / 3 \mathrm{~mL}$ ).

We next divided the patients into subgroups according to their cancer types and pathological stages. The median $\mathrm{FR}+\mathrm{CTC}$ levels in patients with pancreatic cancer, periampullary carcinoma, extrahepatic cholangiocarcinoma (eCCA), HCC, and intrahepatic cholangiocarcinoma (iCCA) were $15.7,11.4,17.7,19.2$, and $20.3 \mathrm{FU} / 3 \mathrm{~mL}$, respectively. For pancreatic cancer, the sensitivity and specificity of 
Table 1 Patients' demographics

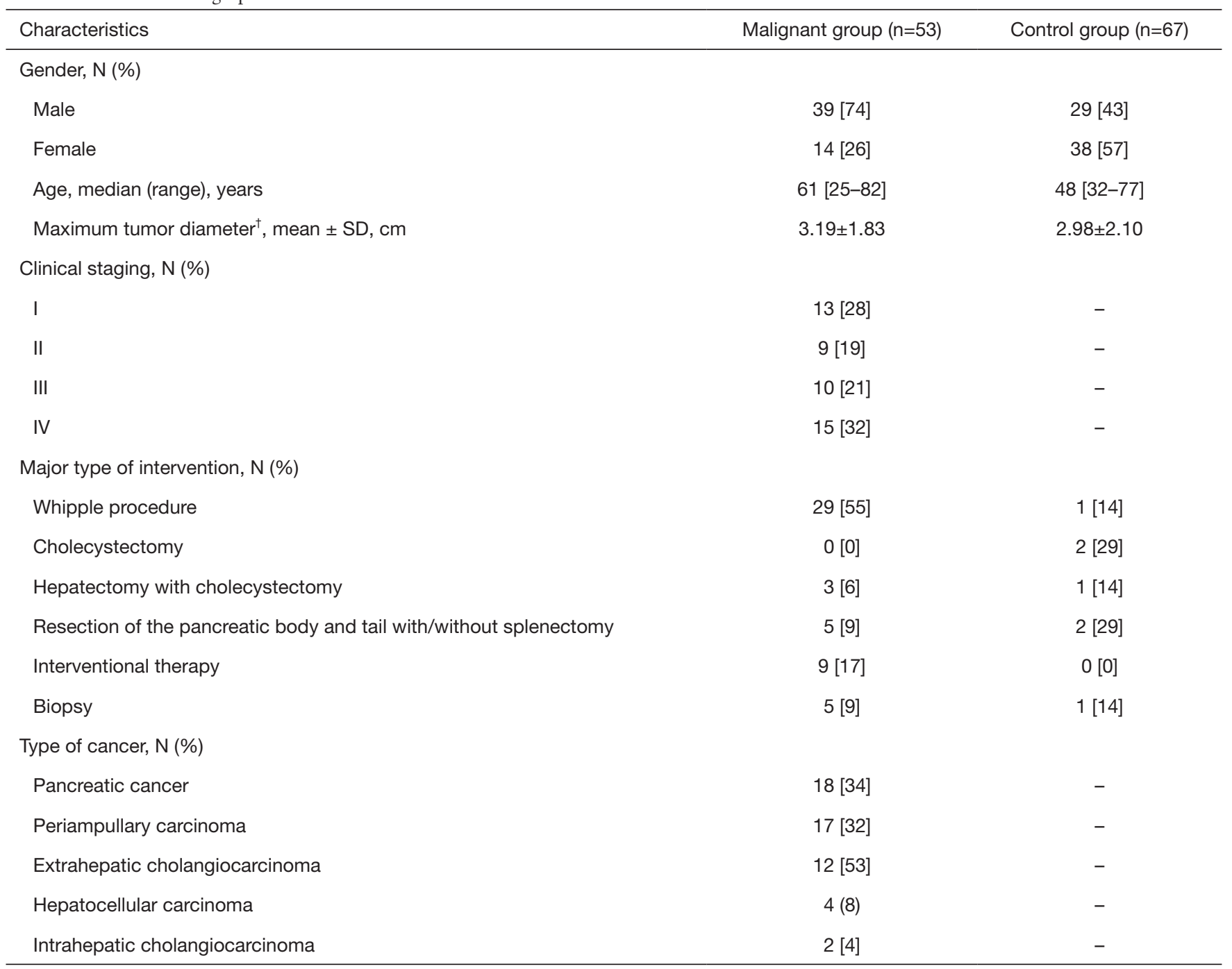

${ }^{\dagger}$, only 47 patients in malignant group and 3 benign diseases patients with suspicious HBPs before surgery had maximum tumor diameter and major type of surgery available. SD, standard deviation.

FR+CTC were $94.4 \%$ and $91.8 \%$, respectively, with $9.65 \mathrm{FU} / 3 \mathrm{~mL}$ as the optimal cut-off value (area under curve $=0.963,95 \%$ CI: 0.925-1.000, Figure 2B). For periampullary carcinoma, the sensitivity and specificity of $\mathrm{FR}+\mathrm{CTC}$ were $100 \%$ and $79.1 \%$, respectively, with $8.65 \mathrm{FU} / 3 \mathrm{~mL}$ as the optimal cut-off value (area under curve $=0.930,95 \%$ CI: $0.877-0.984$ ). For eCCA, the sensitivity and specificity of FR+CTC were $91.7 \%$ and $94.1 \%$, respectively, with $10.35 \mathrm{FU} / 3 \mathrm{~mL}$ as the optimal cut-off value (area under curve $=0.934,95 \%$ CI: $0.833-$ 1.000). For HCC, the sensitivity and specificity of FR+CTC were $100 \%$ and $79.1 \%$, respectively, with $8.7 \mathrm{FU} / 3 \mathrm{~mL}$ as the optimal cut-off value (area under curve $=0.938,95 \%$
CI: 0.849-1.000). For iCCA, the sensitivity and specificity of FR+CTC were $100 \%$ and $98.5 \%$, respectively, with $13.35 \mathrm{FU} / 3 \mathrm{~mL}$ as the optimal cut-off value (area under curve $=0.993,95 \%$ CI: 0.972-1.000). The median FR+CTC levels in patients with early-stage (stage I-II) and latestage HBPs were 11.8 and $19.0 \mathrm{FU} / 3 \mathrm{~mL}$, respectively (Figure 2C). Patients with late-stage HBPs had significantly higher FR+CTC levels compared to those with early-stage diseases $(\mathrm{P}=0.049)$.

In the subgroup of patients with pancreatic cancers, we found that the median FR+CTC level was significantly higher when the tumor was located at the tail compared to that at the head $(22.1$ versus $12.4 \mathrm{FU} / 3 \mathrm{~mL}$, respectively, 
A

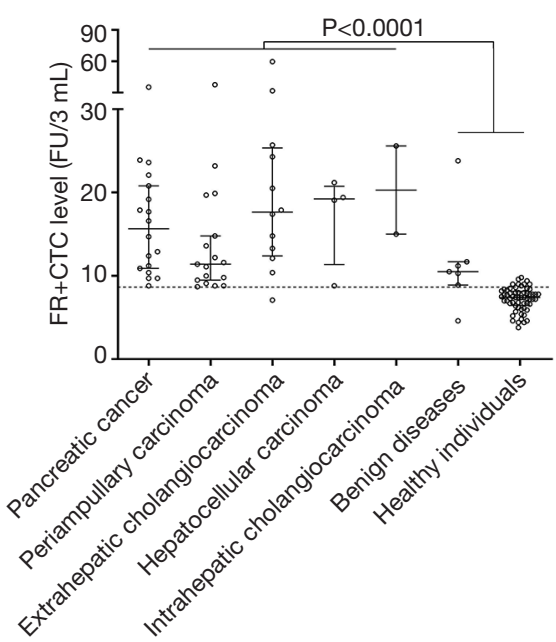

C

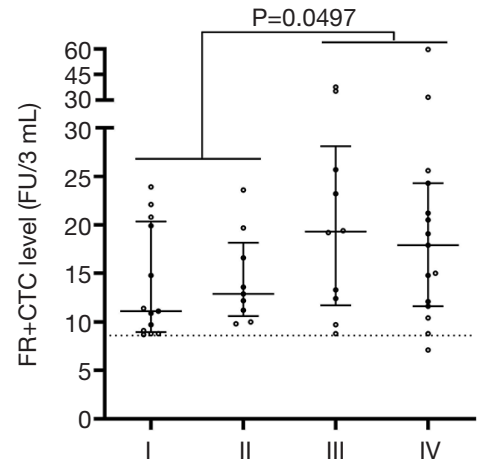

B
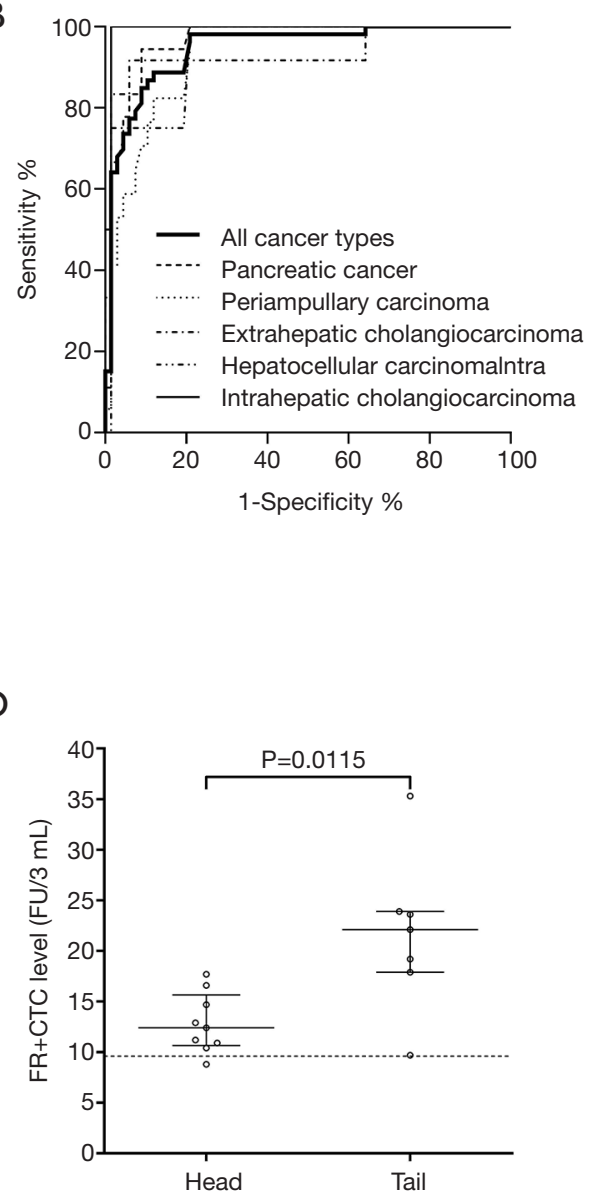

Figure 2 Pre-operative FR+CTC levels. (A) Dot plots showing the FR+CTC levels in patients with different pathological diagnosis. The dotted line represents the cut-off value: $8.65 \mathrm{FU} / 3 \mathrm{~mL}$. (B) ROC curves of different cancer types. (C) Dot plots showing the FR+CTC levels in patients with different pathological stages. (D) Dot plots showing the FR+CTC levels in pancreatic cancer patients with different tumor locations (head: $\mathrm{n}=9$, tail: $\mathrm{n}=7$ ). The dotted line represents the determined cut-off value for pancreatic cancer: $9.65 \mathrm{FU} / 3 \mathrm{~mL}$. FR+CTC, folate receptor-positive circulating tumor cell; ROC, receiver operating characteristic.

$\mathrm{P}=0.012$, Figure 2D).

\section{Comparison with serum biomarkers}

Prior to operation, a subset of the patients concurrently received one or more serum biomarker tests including CA 125, CA 19-9, and CEA. As shown in Table 2, the detection rate of FR+CTC was superior to these routinely used tumor biomarkers for HBPs.

\section{Dynamic change in FR+CTC level}

Of the 53 patients in the malignant group, 16 had matched post-operative FR+CTC results for the dynamic analysis, including 8 patients with pancreatic cancers and 8 with periampullary carcinoma. Post-operative FR+CTC levels were significantly reduced compared to the pre-operative FR+CTC levels (10.4, 95\% CI: $8.3-12.3$ versus $12.3,95 \%$ CI: 9.3-22.9 FU/3 mL, P=0.008, respectively, Figure 3). The average percentage change in FR+CTC level after surgery was $-24.7 \%$. Only four patients showed an increase in FR+CTC level after surgery $(+2.1 \%,+15.9 \%,+21.8 \%$, and $+35.7 \%$, respectively). Post-operative surveillance is still ongoing for these patients.

\section{Discussion}

As recently reported in a nation-wide pooled analysis, 
Table 2 The detection rate of FR+CTC and serum biomarkers

\begin{tabular}{lcc}
\hline Biomarkers & Cut-off value & Detection rate \\
\hline FR+CTC & $8.65 \mathrm{FU} / 3 \mathrm{~mL}$ & $98.1 \%(52 / 53)$ \\
CA 125 & $35 \mathrm{U} / \mathrm{mL}$ & $26.2 \%(11 / 42)$ \\
CA $19-9$ & $37 \mathrm{U} / \mathrm{mL}$ & $50 \%(22 / 44)$ \\
CEA & $5 \mathrm{ng} / \mathrm{mL}$ & $25.6 \%(11 / 43)$ \\
\hline
\end{tabular}

CA125, carbohydrate antigen 125; CA 19-9, carbohydrate antigen 19-9; CEA, carcinoembryonic antigen; FR+CTC, folate receptor-positive circulating tumor cell.

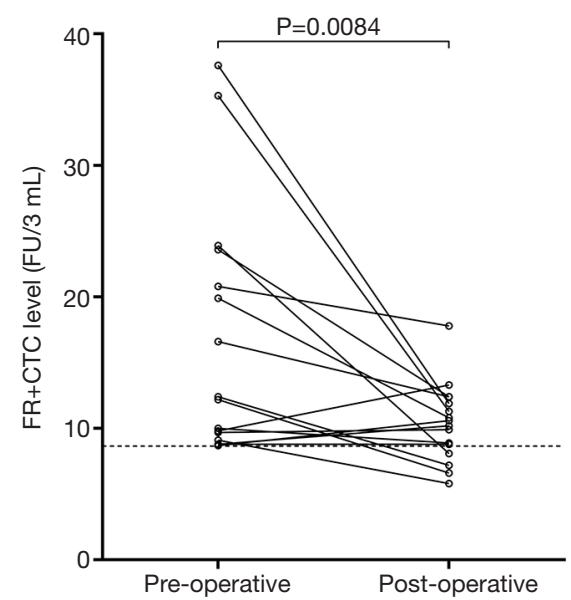

Figure 3 Dynamic change in FR+CTC levels. Dot plot showing the matched pre- and post-operative FR+CTC levels of the 16 patients in the malignant group. The dotted line represents the cut-off value: $8.65 \mathrm{FU} / 3 \mathrm{~mL}$. FR+CTC, folate receptor-positive circulating tumor cell.

the survival rate of most of the cancer types have been gradually improving in China partly due to the initiation of cancer screening and early detection programs, except for the more lethal cancer types such as pancreatic and gallbladder cancers (37). Therefore, to better diagnose and manage HBPs, novel and more effective biomarkers are urgently needed. In this preliminary study, we sought to evaluate FR+CTCs as a non-invasive biomarker for HBPs. Our results suggested that FR+CTC could be a potential candidate in the setting of differential diagnosis and surveillance.

For the differential diagnosis of HBPs, FR+CTC showed a high sensitivity (98.1\%) and specificity (79.1\%). Further, when each cancer type is analyzed individually, FR+CTC showed adequate sensitivity and specificity in each of the
5 types: pancreatic cancer, periampullary carcinoma, eCCA, HCC, and iCCA. Compared to routine serum tumor biomarkers such as CA-125, CA-199, and CEA, FR+CTC exhibited an excellent detection rate. These results suggested that FR+CTC could be an efficacious biomarker in identifying HBPs. In general, the false-positive rate of FR+CTC was greater than $10 \%$, suggesting that its use in routine cancer screening should be adopted with caution. Nevertheless, for particular cancer types such as pancreatic cancers, eCCA and iCCA, our results demonstrated that the specificities were superior $(91.8 \%, 94.1 \%$ and $98.5 \%$, respectively), implying that FR+CTC test could be potentially applied for cancer screening in these cancer types. Additional large studies are required to validate these findings.

It should be noticed that the 6 patients with benign inflammatory diseases in this study showed false-positive $\mathrm{FR}+\mathrm{CTC}$ results. One possible explanation is that the elevated white blood cell count in inflammatory diseases patients caused an insufficient depletion of leukocytes, resulting in the non-specific labelling of detection probes and the false-positive FR+CTC results. However, because of the small number of benign patients recruited in this study, the effect of inflammatory diseases on false positivity of $\mathrm{FR}+\mathrm{CTC}$ remains to be evaluated. The combination of FR+CTC with CA 19-9, which has been proven to be useful in differentiating pancreatic cancer and inflammatory diseases (38), can potentially rectify the concerns for false positivity.

Furthermore, the dynamic change of $\mathrm{FR}+\mathrm{CTC}$ preand post-operation was consistent with reduction of tumor burden. For the 16 patients with paired pre- and postoperative FR+CTC test, an average percentage decrease of $-23.8 \%$ in $\mathrm{FR}+\mathrm{CTC}$ level was observed, suggesting that surgery was effective to remove the source of the CTCs. Such a change may be further used to monitor the risk of the disease recurrence. Previous studies demonstrated that a decrease in CTC count after therapy is associated with better prognosis in non-small cell lung cancer (39). Recently, Shen et al. also reported that the reduction of FR+CTC after first-line chemotherapy is a prognostic biomarker for patients with small cell lung cancer. The prognostic value of preoperative FR+CTC and its dynamic changes in the HBP patients receiving surgical treatment remains to be explored. Follow-up of the recruited patients is ongoing in this study.

Interestingly, we found that the FR+CTC level in pancreatic cancer was significantly higher when the tumor 
was located at the tail compared to that at the head. A previous study reported that pancreatic cancers of the tail were associated with a significantly higher rate of metastatic diseases $(67 \%$ versus $36 \%, \mathrm{P}<0.001)$. Since metastatic cancers possess a higher tumor load, it is possible that they tend to release more CTCs into the blood circulation (28-31).

The major limitation of the current study is the small sample size, especially for the subgroup of benign diseases where only 7 patients with inflammatory diseases were enrolled. As such, results from the present study require further validation. Nonetheless, our exploratory study, suggested that FR+CTC could be a potential biomarker for the differential diagnosis and surveillance in HBPs and further studies can be conducted to fully explore the clinical significance of FR+CTC in HBPs.

\section{Acknowledgments}

We thank Genosaber Biotech Co. Ltd. for providing technical support in this study.

Funding: This study was supported by hospital research fund (general project) from The Second Affiliated (Xinqiao) Hospital of Army Medical University (Project No.: 2016YLC18; Funding No.: 2016D390).

\section{Footnote}

Reporting Checklist: The authors have completed the STARD reporting checklist. Available at http://dx.doi.org/10.21037/ tcr-20-2192

Data Sharing Statement: Available at http://dx.doi. org/10.21037/tcr-20-2192

Conflicts of Interest: All authors have completed the ICMJE uniform disclosure form (available at http://dx.doi. org/10.21037/tcr-20-2192). The authors have no conflicts of interest to declare.

Ethical Statement: The authors are accountable for all aspects of the work in ensuring that questions related to the accuracy or integrity of any part of the work are appropriately investigated and resolved. The study was conducted in accordance with the Declaration of Helsinki (as revised in 2013). The Institutional Review Board of Xinqiao Hospital, Army Medical University and Renmin Hospital of Wuhan University have approved the study protocol (AF/
SC-08/1.0). All patients enrolled completed the informed consent form.

Open Access Statement: This is an Open Access article distributed in accordance with the Creative Commons Attribution-NonCommercial-NoDerivs 4.0 International License (CC BY-NC-ND 4.0), which permits the noncommercial replication and distribution of the article with the strict proviso that no changes or edits are made and the original work is properly cited (including links to both the formal publication through the relevant DOI and the license). See: https://creativecommons.org/licenses/by-nc-nd/4.0/.

\section{References}

1. Bray F, Ferlay J, Soerjomataram I, et al. Global cancer statistics 2018: GLOBOCAN estimates of incidence and mortality worldwide for 36 cancers in 185 countries. CA Cancer J Clin 2018;68:394-424.

2. Siegel RL, Miller KD, Jemal A. Cancer Statistics, 2017. CA Cancer J Clin 2017;67:7-30.

3. Omata M, Cheng AL, Kokudo N, et al. Asia-Pacific clinical practice guidelines on the management of hepatocellular carcinoma: a 2017 update. Hepatol Int 2017;11:317-70.

4. Department of Medical Administration NHaHCotPsRoC. Guidelines for diagnosis and treatment of primary liver cancer in China (2019 edition). Zhonghua Gan Zang Bing Za Zhi 2020;28:112-28.

5. Doherty B, Nambudiri VE, Palmer WC. Update on the Diagnosis and Treatment of Cholangiocarcinoma. Curr Gastroenterol Rep 2017;19:2.

6. Puleo F, Marechal R, Demetter P, et al. New challenges in perioperative management of pancreatic cancer. World J Gastroenterol 2015;21:2281-93.

7. Bruix J, Sherman M. Management of hepatoceullular carcinoma. Hepatology 2005;42:1208-36.

8. Oettle H, Post S, Neuhaus P, et al. Adjuvant chemotherapy with gemcitabine vs observation in patients undergoing curative-intent resection of pancreatic cancer - A Randomized controlled trial. JAMA 2007;297:267-77.

9. McGuigan A, Kelly P, Turkington RC, et al. Pancreatic cancer: A review of clinical diagnosis, epidemiology, treatment and outcomes. World J Gastroenterol 2018;24:4846-61.

10. Ilie M, Hofman V, Long-Mira E, et al. "Sentinel" Circulating Tumor Cells Allow Early Diagnosis of Lung Cancer in Patients with Chronic Obstructive Pulmonary 
Disease. PLoS One 2014;9:e111597.

11. Pantel K, Alix-Panabieres C. Circulating tumour cells and cell-free DNA in gastrointestinal cancer. Nat Rev Gastroenterol Hepatol 2017;14:73-4.

12. Chen C, Ke J, Zhou XE, et al. Structural basis for molecular recognition of folic acid by folate receptors. Nature 2013;500:486-9.

13. Nunez MI, Behrens C, Woods DM, et al. High Expression of Folate Receptor Alpha in Lung Cancer Correlates with Adenocarcinoma Histology and Mutation. J Thorac Oncol 2012;7:833-40.

14. He W, Wang H, Hartmann LC, et al. In vivo quantitation of rare circulating tumor cells by multiphoton intravital flow cytometry. Proc Natl Acad Sci U S A 2007;104:11760-5.

15. Nunez MI, Behrens C, Woods DM, et al. High expression of folate receptor alpha in lung cancer correlates with adenocarcinoma histology and EGFR [corrected] mutation. J Thorac Oncol 2012;7:833-40.

16. Zhang Z, Wang J, Tacha DE, et al. Folate receptor $\alpha$ associated with triple-negative breast cancer and poor prognosis. Arch Pathol Lab Med 2014;138:890-5.

17. Parker N, Turk MJ, Westrick E, et al. Folate receptor expression in carcinomas and normal tissues determined by a quantitative radioligand binding assay. Anal Biochem 2005;338:284-93.

18. He W, Kularatne SA, Kalli KR, et al. Quantitation of circulating tumor cells in blood samples from ovarian and prostate cancer patients using tumor-specific fluorescent ligands. Int J Cancer 2008;123:1968-73.

19. Chen X, Zhou F, Li X, et al. Folate Receptor-Positive Circulating Tumor Cell Detected by LT-PCR-Based Method as a Diagnostic Biomarker for Non-Small-Cell Lung Cancer. J Thorac Oncol 2015;10:1163-71.

20. Lou J, Ben S, Yang G, et al. Quantification of rare circulating tumor cells in non-small cell lung cancer by ligand-targeted PCR. PLoS One 2013;8:e80458.

21. Lian H, Ding Z, Yuan D, et al. Diagnostic Value of Folate Receptor-positive Circulating Tumor Cell in Lung Cancer: A Pilot Study. Zhongguo Fei Ai Za Zhi 2016;19:813-20.

22. Qi F, Liu Y, Zhao R, et al. Quantitation of rare circulating tumor cells by folate receptor $\alpha$ ligand-targeted PCR in bladder transitional cell carcinoma and its potential diagnostic significance. Tumour Biol 2014;35:7217-23.

23. Cheng H, He W, Yang J, et al. Ligand-targeted polymerase chain reaction for the detection of folate receptor-positive circulating tumour cells as a potential diagnostic biomarker for pancreatic cancer. Cell Prolif 2020;53:e12880.
24. Li N, Zhong D, Chen H, et al. The utility of folate receptor-positive circulating tumor cell in cancer diagnosis in the elderly population. Cancer Manag Res 2019;11:4097-107.

25. Shen J, Zhao J, Jiang T, et al. Predictive and prognostic value of folate receptor-positive circulating tumor cells in small cell lung cancer patients treated with first-line chemotherapy. Oncotarget 2017;8:49044-52.

26. Wang L, Wu C, Qiao L, et al. Clinical Significance of Folate Receptor-positive Circulating Tumor Cells Detected by Ligand-targeted Polymerase Chain Reaction in Lung Cancer. J Cancer 2017;8:104-10.

27. Yu Y, Chen Z, Dong J, et al. Folate receptor-positive circulating tumor cells as a novel diagnostic biomarker in non-small cell lung cancer. Transl Oncol 2013;6:697-702.

28. Wang L, Wu C, Qiao L, et al. Clinical Significance of Folate Receptor-positive Circulating Tumor Cells Detected by Ligand-targeted Polymerase Chain Reaction in Lung Cancer. J Cancer 2017;8:104-10.

29. Chen X, Zhou F, Li X, et al. Folate Receptor-Positive Circulating Tumor Cell Detected by LT-PCR-Based Method as a Diagnostic Biomarker for Non-Small-Cell Lung Cancer. J Thorac Oncol 2015;10:1163-71.

30. Lou J, Ben S, Yang G, et al. Quantification of Rare Circulating Tumor Cells in Non-Small Cell Lung Cancer by Ligand-Targeted PCR. PLoS One 2013;8:e80458.

31. Yu Y, Chen Z, Dong J, et al. Folate Receptor-Positive Circulating Tumor Cells as a Novel Diagnostic Biomarker in Non-Small Cell Lung Cancer. Transl Oncol 2013;6:697702 .

32. D'Angelica M, Ammori J, Gonen M, et al. Folate receptor-alpha expression in resectable hepatic colorectal cancer metastases: patterns and significance. Mod Pathol 2011;24:1221-8.

33. Shen J, Hu Y, Putt KS, et al. Assessment of folate receptor alpha and beta expression in selection of lung and pancreatic cancer patients for receptor targeted therapies. Oncotarget 2018;9:4485-95.

34. Cai L, Michelakos T, Ferrone CR, et al. Expression status of folate receptor alpha is a predictor of survival in pancreatic ductal adenocarcinoma. Oncotarget 2017;8:37646-56.

35. Fan W, Xu Y, Li Z, et al. Folic acid-modified $\beta$-cyclodextrin nanoparticles as drug delivery to load DOX for liver cancer therapeutics. Soft Mater 2019;17:437-47.

36. Omote S, Takata K, Tanaka T, et al. Overexpression of folate receptor alpha is an independent prognostic factor for outcomes of pancreatic cancer patients. Med Mol 
Morphol 2018;51:237-43.

37. Zeng $\mathrm{H}$, Chen W, Zheng R, et al. Changing cancer survival in China during 2003-15: a pooled analysis of 17 population-based cancer registries. Lancet Glob Health 2018;6:E555-67.

38. Safi F, Roscher R, Bittner R, et al. High sensitivity and specificity of CA 19-9 for pancreatic carcinoma

Cite this article as: $\mathrm{He}$ YG, Zheng L, Gao MF, Tang YC, Li YM, Yu KH, Li J, Huang XB. A preliminary study of the clinical significance of folate receptor-positive circulating tumor cell in the management of hepatobiliary-pancreatic cancers. Transl Cancer Res 2020;9(11):6700-6709. doi: 10.21037/tcr-202192 in comparison to chronic pancreatitis. Serological and immunohistochemical findings. Pancreas 1987;2:398-403.

39. Liu Y, Yuan D, Ye W, et al. Prognostic value of circulating endothelial cells in non-small cell lung cancer patients: a systematic review and meta-analysis. Transl Lung Cancer Res 2015;4:610-8. 